\title{
Reproducibility of single protein explosions induced by X-ray lasers ${ }^{\dagger}$
}

\begin{abstract}
Christofer Östlin, ${ }^{a}$ Nicuşor Tîmneanu, ${ }^{a, b} \mathrm{H}$. Olof Jönsson, ${ }^{a}$ Tomas Ekeberg, ${ }^{c}$ Andrew V. Martin, ${ }^{d, e}$ and Carl Caleman $* a, c$

Single particle imaging (SPI) using X-ray pulses has become increasingly attainable with the advent of high-intensity free electron lasers. Eliminating the need for crystallized samples enables structural studies of molecules previously inaccessible by conventional crystallography. While this emerging technique already demonstrates substantial promise, some obstacles need to be overcome before SPI can reach its full potential. One such problem is determining the spatial orientation of the sample at the time of X-ray interaction. Existing solutions rely on diffraction data and are computationally demanding and sensitive to noise. In this in silico study, we explore the possibility of aiding these methods by mapping the ion distribution as the sample undergoes a Coulomb explosion following the intense ionization. By detecting the ions ejected from the fragmented sample, the orientation of the original sample should be possible to determine. Knowledge of the orientation has been shown earlier to be of substantial advantage in the reconstruction of the original structure. 150 explosions of each of twelve separate systems - four polypeptides with different amounts of surface bound water - were simulated with molecular dynamics (MD) and the average angular distribution of carbon and sulfur ions was investigated independently. The results show that the explosion maps are reproducible in both cases, supporting the idea that orientation information is preserved. Additional water seems to restrict the carbon ion trajectories further through a shielding mechanism, making the maps more distinct. For sulfurs, water has no significant impact on the trajectories, likely due to their higher mass and greater ionization cross section, indicating that they could be of particular interest. Based on these findings, we conclude that explosion data can aid spatial orientation in SPI experiments and could substantially improve the capabilities of the novel technique.
\end{abstract}

\section{Introduction}

With its introduction in the early $20^{\text {th }}$ century, X-ray crystallography revolutionized science by providing the means to observe the structures of molecules, most notably those of biologically important proteins. Since then, the method has been used to solve a vast majority of the protein structures known today according to the Protein Data Bank, http://www.rcsb.org/pdb. ${ }^{1}$ Despite its undeniable success, X-ray crystallography is not without drawbacks, and initiatives have been taken to circumvent the perhaps most glaring of these limitations; the intrinsic need of crystals.

Utilizing an X-ray free-electron laser (XFEL) may enable the collection of structure-revealing diffraction patterns from single molecules through its intense and ultrashort pulses - a technique known as diffraction before destruction ${ }^{2}$. The method has been used with larger samples to produce high-quality diffraction patterns and full three-dimensional reconstructions at the Linac Coherent Light Source (LCLS) in California, ${ }^{3,4}$ and a roadmap has been established to guide the future development of single particle imaging at XFELs. ${ }^{5}$ While it eliminates the dependence on a crystalline sample, the severe radiation damage invariably causes the sample to explode. For this reason, a new, identical sample needs to be introduced into the beam for every diffraction pattern recorded. Assuming no preorientation, each of the samples will be in a different and unknown spatial orientation. To record a full dataset covering all angles so that the three-dimensional

\footnotetext{
${ }^{a}$ Department of Physics and Astronomy, Uppsala University, Box 516, SE-751 20 Uppsala, Sweden

${ }^{b}$ Department of Cell and Molecular Biology, Uppsala University, Box 596, SE-751 24 Uppsala, Sweden

${ }^{c}$ Center for Free-Electron Laser Science, DESY, Notkestraße 85, DE-22607 Hamburg, Germany

${ }^{d}$ ARC Centre of Excellence for Advanced Molecular Imaging, School of Physics, University of Melbourne, Parkville, Victoria 3010, Australia

e School of Science, RMIT University, Melbourne, Victoria 3001, Australia

*E-mail: carl.caleman@physics.uu.se

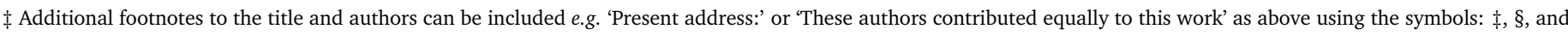

ๆ. Please place the appropriate symbol next to the author's name and include a $\backslash$ footnotetext entry in the the correct place in the list.
} 
a. SPI: random orientation

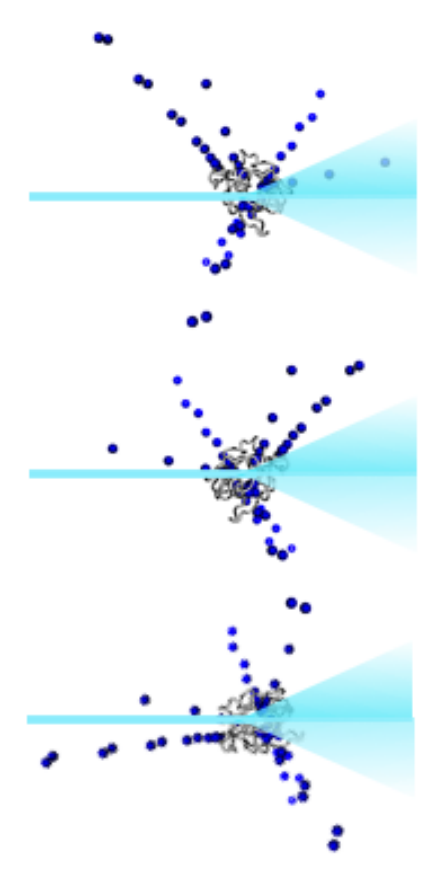

b. Images from unknown orientation

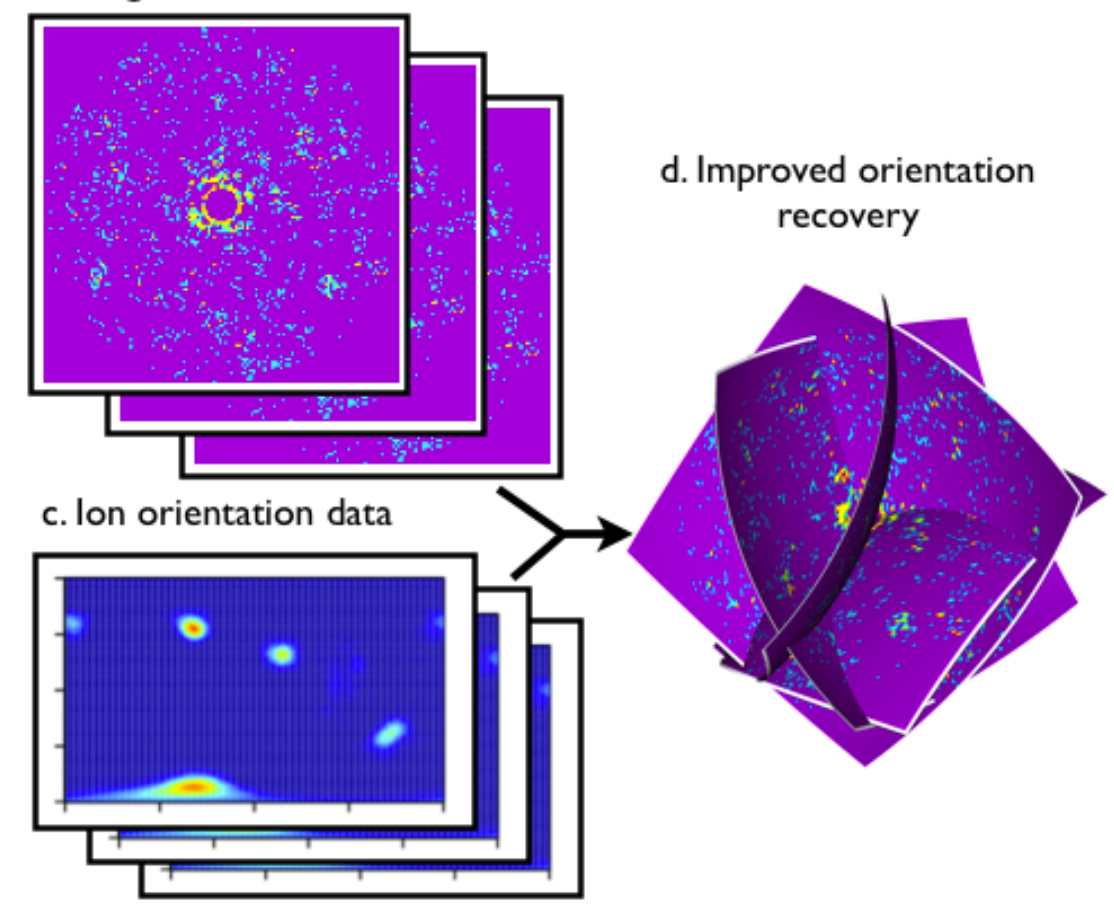

Fig. 1 From explosions to image orientation recovery. a. Each sample is hit by an X-ray pulse in an unknown orientation, generating a diffraction pattern and ion distribution map. The blue spheres are the sulfur ions and are illustrated as trajectories over 9 time steps. The rest of the protein, in grey, is only shown in its initial conformation. Diffraction patterns (b.) and ion maps (c.) are simultaneously recorded without prior knowledge of the sample's orientation. d. By incorporating orientation information present in ion data, algorithms can potentially assemble a more accurate 3D diffraction dataset, which is the input for structure determination methods.

structure can be reconstructed, hundreds of diffraction patterns needs to be collected. ${ }^{4}$

Orienting and assembling all the various diffraction images remains a cumbersome task if the sample is delivered randomly. ${ }^{4}$ In conventional crystallography, all patterns are collected from the same crystal as it is being rotated, completely retaining the relative orientation data. But when imaging single particles one instead needs to retrieve this data post experimentally, a process referred to as "orientation recovery". Algorithms have been developed that can perform this task, such as the so called Expand, Maximize, and Compress (EMC) algorithm ${ }^{6}$. However, they are not always able to recover the correct original orientation due to, for example, background scattering and sample heterogeneity. While sample heterogeneity for instance can be addressed by classification or manifold data analysis methods, ${ }^{5,7}$ an experimental technique to determine the orientation would mitigate the sources of noise in the diffraction. The reduction in computational time would consequently improve algorithm performance significantly, allowing for structural studies to make much better use of data from current and future XFELs. Figure 1 illustrates how the addition of ion data would improve the assembly of diffracted images and the orientation recovery.

In this theoretical study, we explore an alternative approach to solving this problem; the feasibility of determining sample orientation based on its explosion pattern. The high intensity of the XFEL pulse causes many of the electrons of the sample to be stripped away within tens of femtoseconds. The remaining ions are consequently exposed to Coulomb forces causing the sample to explode. ${ }^{8-10}$ If such an XFEL-induced Coulomb explosion exhibits a high level of reproducibility, it is reasonable to infer that acquiring the ion explosion pattern would aid in orientation determination. Instead of relying only on the diffraction pattern obtained by detecting the photons of the pulse alone, one could also detect the ejected ion fragments long after the interaction event has taken place. This information could then be used to enhance the accuracy and reduce the time required to assemble 3D datasets of single particles. The idea has been evaluated before using a simplified model sample with promising results, ${ }^{11}$ and here we extend the methodology to proteins of known structures with and without encapsulating water. 


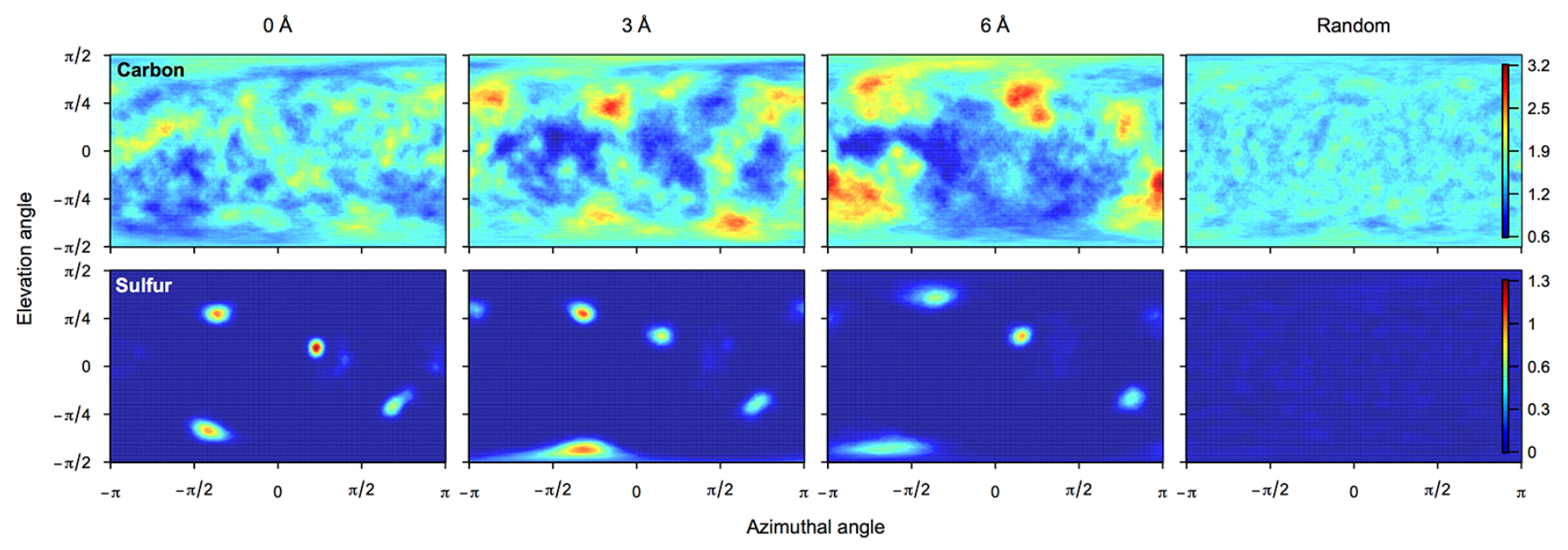

Fig. 2 Spherical distribution of carbon [top] and sulfur [bottom] ions from Coulomb explosions. Shown are lysozyme samples with three water coverages and a random control. The intensity of the XFEL pulse is $10^{12}$ photons at $8 \mathrm{keV}$ over a $100 \mathrm{~nm}$ diameter focal spot. The ion density maps are shown as heatmaps of the bin mean counts for each system. All of them show signs of "hot spots" and "cold spots" - areas of significantly higher or lower ion densities. The clear spots in the bottom panels indicate consistent flight paths of the sulfur ions and therefore highly reproducible explosions. By comparison, the last column shows how similar maps based on the same number of explosions (150) would look if all the measured ions were distributed uniformly over the sphere $(i . e$. fully irreproducible). The differences between the sample maps and random controls suggest that explosion patterns are reproducible and that some orientation information could be retrieved. Color scales indicate the average ion count over all simulations.

\section{Method}

Four different polypeptide systems of known structure, shown in Figure 1 in Supplementary Information, were chosen for this in silico study. These were, with Protein Data Bank structure IDs given in brackets: the 20-residue miniprotein construct Trp-cage (1L2Y) ${ }^{12}$; the C-terminal portion of L7/L12 found in bacterial ribosomes (1CTF) ${ }^{13}$; the human regulatory protein ubiquitin $(1 \mathrm{UBI})^{14}$; and the hen egg white enzyme lysozyme (1AKI) ${ }^{15}$. The structural stability of these particular molecules in vacuum, henceforth referred to as Trp, Ctf, Ubi and Lys respectively, were previously studied by van der Spoel and coworkers. ${ }^{16,17}$ In these studies, apart from using dry versions of the samples, they also encapsulated each protein in water layers corresponding to thicknesses of $3 \AA$ and $6 \AA$. Excess water was then allowed to evaporate while remaining water molecules arranged themselves energetically favorably around the sample, resulting in a total surface water coverage of approximately $60 \%$ and $70 \%$ respectively. This was done through molecular dynamics (MD) vacuum simulations and the resulting protein/water complexes, along with the waterless variants, from Marklund et al. ${ }^{17}$ were adopted for the current work.

\subsection{Simulations}

Similarly to previous studies, ${ }^{2,10,18-20}$ all MD simulations were carried out with the GROMACS software suite ${ }^{21}$ using the OPLS-AA force field, ${ }^{22,23}$ and data collection consisted of two steps termed the presimulations and the explosions. In the former, the different protein/water combinations underwent an additional nanosecond of vacuum simulations using $1 \mathrm{fs}$ time steps, during which small structural variations occurred. The spatial orientation was kept unchanged by precluding translational and rotational motion of the protein, also ensuring negligible movement of the protein center of mass. This restriction was not applied to solvent molecules since only the protein orientation is of interest. The generated trajectories consisted of a large number of slightly different variations, all of which can be considered realistic in the setting of a single particle imaging experiment utilizing a sample delivery system that forms aerosols such as a gas dynamic virtual nozzle $(\mathrm{GDVN})^{24,25}$ and an aerodynamic lens stack. ${ }^{26} \mathrm{~A}$ random frame from these trajectories was then chosen as a starting structure for each of the explosion simulations.

Some alterations to the chosen frame were implemented to model sample dynamics in an XFEL pulse. These included the modification of the TIP4P water model ${ }^{27}$ by removing the massless fourth dummy particles and the resetting of the 
Lennard-Jones parameters. This would cause atoms to be displaced over time, but ionization occurs on timescales much shorter than atomic movement driven by Coulomb forces, such that no significant displacement of the atoms will have time to manifest before the onset of the X-ray pulse. We also incorporated Morse potentials ${ }^{28}$ instead of harmonic bonds to allow for the simulation of bond breaking.

Exposure to an XFEL pulse was then simulated through the GROMACS built-in ionize function with a randomized ionization sequence. The code simulates an even distribution of photons over the focus and tracks both photoionization events and Auger processes. Photoionization is treated as a stochastic interaction between X-ray photons and atoms, assuming unpolarized X-rays and homogeneous spatial distribution of the free electrons. Primary ionization probabilities are determined from element-specific cross sections, which are well known. Parameters were set to reflect those of current and future XFELs - i.e. the Coherent X-ray Imaging (CXI) ${ }^{29}$ end station at the LCLS - with an intensity of $10^{12}$ photons, a photon energy of $8 \mathrm{keV}(\lambda=1.55 \AA)$ and a beam focus diameter of $100 \mathrm{~nm} .{ }^{29}$ This beam size is approximately one order of magnitude greater than the diameter of Lys, the largest of the samples, thus ensuring full beam immersion of all samples. Lastly, the temporal profile of the pulse was simulated as Gaussian with a FWHM of 50 fs to represent an average SASE-generated pulse. ${ }^{30}$ For our small samples in vacuum, released electrons are assumed to leave the sample and do not contribute to the simulated electrostatic forces. In a large sample, the ejected electrons would scatter on the surrounding valence electrons causing secondary ionizations, a process that occurs within the duration of the X-ray pulse ${ }^{20}$. The largest molecule simulated here is around $50 \AA$ across, a distance in which it is less likely that either the ejected photoelectrons or the Auger electrons will interact with the surrounding electrons ${ }^{19}$. As the electrons leave, a net charge will build up in the sample, which at some point will lead to trapping of escaping electrons ${ }^{9}$ and increase the likelihood of further ionizations. Our model does not capture this phenomenon, meaning that the ionization level might be slightly higher towards the end of the pulse than our model estimates. By comparison, our model predicts the calculated mean ionization levels for Lys, for instance, to be $\sim 1.5$ ionization events per atom after X-ray exposure.

A total of $M=150$ explosions were generated for each combination of four proteins (Trp, Ctf, Ubi, Lys) and three water coverages ( $0 \AA$, $3 \AA, 6 \AA$ ), using 50 attosecond time steps over a total simulation time of 1 ps per sample. At the end the simulations the Coulomb interactions have become weak and the change in ion velocity vectors are negligible. From this point, the final position and velocity vectors of all individual atoms are used to project each of the exploding particles onto a sphere centered in the simulation box. The center of the sphere coincides with the initial center of mass of the protein as established in the presimulations. This generates a full set of ion flight angular coordinates used in the data evaluation.

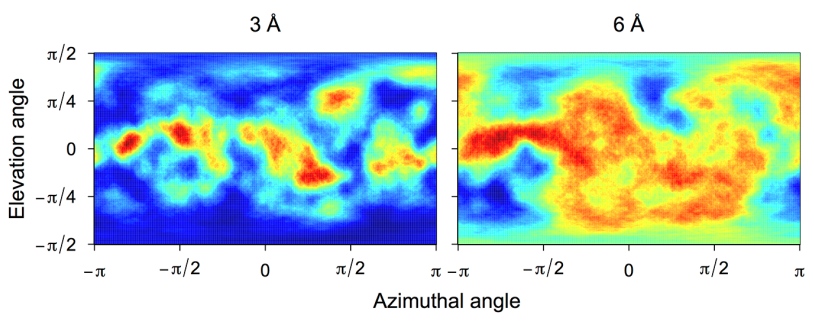

Fig. 3 Ion distributions of solvent oxygens for lysozyme. The average distribution of the oxygen ions originating from surfacebound water molecules. These are complementary to the carbon maps, Figure 2, indicating that the water restricts the possible carbon trajectories. The color scale here is in arbitrary units to give a qualitative illustration of the different distributions, where signal strength ranges from blue (low) to red (high).

\subsection{Analysis}

A binning methodology was adopted to investigate the reproducibility of the explosions. $\mathrm{N}$ different bins were distributed along the surface of the sphere by dividing both of the spherical angle ranges into subintervals of equal length. This generated a 500x250 grid on the sphere where each vertex corresponded to a bin center point. The 125,000 bins would then be defined as spherical caps of a specific size. The size was given by the orthodromic radius of the bins - the greatcircle distance from its center to its border - denoted by $r$. An ejected ion was considered an element of a bin if its position coincided with the cap of the corresponding bin. In other words, since the great-circle distance $d_{i j}$ between the center 
point $i$ of an arbitrary bin and a projected ion $j$ is given by

$$
d_{i j}=\cos ^{-1}\left(\sin \varphi_{i} \sin \varphi_{j}+\cos \varphi_{i} \cos \varphi_{j} \cos \left(\theta_{i}-\theta_{j}\right)\right)
$$

in spherical coordinates, $j$ belongs to the bin centered around $i$ if $d_{i j}<r$. The variables $\theta_{i}, \varphi_{i}$ and $\theta_{j}, \varphi_{j}$ are the azimuthal and polar coordinates of the center point and the ion respectively, and their value ranges are $-\pi<\theta \leq \pi$ and $-\pi / 2 \leq$ $\varphi \leq \pi / 2$. The latter is commonly referred to as elevation angle, which is the terminology we will be using henceforth. A visual explanation of the geometry and binning method can be seen in the Supplementary Information, Figure 2. In our analysis, we chose $r=0.1$ which gives bin areas of roughly $0.25 \%$ of the total sphere area. At 125,000 bins this creates an effective detector area 300 times that of the sphere surface. Note that bins closer to the poles are more densely packed due to spherical geometry.

After binning a selected species of ions, the individual bin count mean values over all simulations were calculated. These average values, along with the bin positions, translates to an angular ion density map highlighting any recurring spatial features of the explosion; an average explosion footprint of the sample.

\section{Results}

We display the footprints as 2D-heatmaps of the registered bin values where axes represent the two spherical angles and analyze them visually. This equirectangular projection of the sphere is similar to how the world map is usually visualized in a standard atlas. Figure 2 shows such maps of carbon and sulfur ion densities of exploding Lys with different water coverage.

First a few words on the method of projection itself, because while it is simple and illustrative it also has its drawbacks. Most notable is the elevation-dependent area distortion. Each horizontal line of pixels in the heatmap correspond to bin values along a circle of latitude of the detector sphere. While being depicted with equal length in the 2D-plot, of course the circumference of those circles along the sphere decreases closer to the poles. This means that the projection will be stretched to varying degrees for the rectangular shape to be preserved. This is the very same reason our bin density is higher at elevations closer to the extremes. So essentially features near the poles will be more "smoothened" and take up a bigger area in the 2D-image than on the sphere. The distortion is a minor problem here, however, as we are mainly interested in identifying ion density hot spots on the sphere rather than measuring them.

For a set of fully random explosions of a sample $-i$. e. where no reproducibility is displayed - one would detect on average the same number of counts in all of the bins, resulting in a highly monochromatic heatmap lacking hot spots of higher or lower ion density. Such random explosion maps were also generated for comparison purposes and are included in the previously mentioned figure. This was done by uniformly distributing the same number of points as detected in each sample on the sphere $\mathrm{M}$ separate times and calculating the individual bin count mean values as before. Therefore, they give an idea of what the various explosion maps would look like in case the explosions were irreproducible.

From Figure 2 it is evident that the displayed explosion maps deviate from the random explosions, some more than others. This holds for all the twelve systems simulated (see Figure 3, Supplementary Information). Because the ion distribution is not uniform it must indeed reflect a certain level of explosion reproducibility. The level seems highly dependent on the system in question though, as the number of ion hot spots and their intensities varies greatly between them. A significant factor affecting the reproducibility across all proteins, however, seems to be the amount of water surrounding it. In the case of the waterless samples one could question if the carbon ion density map even could be separated from a randomized one practically. But, as the number of solvent molecules increases, the carbon ions tend to get concentrated to a smaller area of the detector sphere, resulting in considerably more distinct patterns. The reason for this is likely a sort of shielding; the positioning of the waters around the molecules is fairly static and will consistently block possible ion escape trajectories present in the dry version of the sample. This is supported by Figure 3 (and Figure 4 in Supplementary Information), which shows the detector data for solvent oxygen ions of the water-covered cases (that is, oxygens belonging to the protein chain are not included). The patterns are almost perfect inversions of the carbon counterparts indicating that carbons go one way, oxygens another, and upon adding more water the carbons will be further limited in their trajectories.

In contrast, this does not seem to be the case for the sulfur ions. From the heatmaps in Figure 2 we observe both the deviation from randomness as noted with carbon, and a lesser impact, if any, by the solvent molecules. The distinct spots remain largely unchanged both in terms of intensities and positions regardless of water content. This is promising 
Table 1 Fraction of detector sphere where the ion signal is statistically significant $\left(\left|I_{p}-\mu_{r}\right|>5 \sigma_{r}\right)$ in lysozyme. Carbon and sulfur for the various water shells are shown.

\begin{tabular}{ccc} 
Element & Water layer & Fraction of sphere \\
\hline $\mathrm{C}$ & $0 \AA$ & 0.02 \\
$\mathrm{C}$ & $3 \AA$ & 0.08 \\
$\mathrm{C}$ & $6 \AA$ & 0.13 \\
$\mathrm{~S}$ & $0 \AA$ & 1.0 \\
$\mathrm{~S}$ & $3 \AA$ & 0.98 \\
$\mathrm{~S}$ & $6 \AA$ & 0.96
\end{tabular}

for orientation determination since the water encapsulation profile is subject to variation under experimental conditions. Moreover, the sulfur content of proteins is lower than that of other elements, making it considerably easier to distinguish between the different individual atoms. With lesser data points to fit, the space of possible orientations would be smaller and an algorithm developed to find the best fit would consequently be faster.

One way of quantizing the significance of the hot spots is to determine the mean $\mu_{r}$ and standard deviation $\sigma_{r}$ of the random signal and compare the intensities of the protein signal to those. Measured signal outside a set threshold from the mean, for instance $5 \sigma_{r}$, can then be considered statistically meaningful. The fraction of the sphere showing significant signal gives a measure by which the different samples can be compared. Let $I_{r}(\theta, \varphi)$ be the heatmap of the random distribution and $I_{p}(\theta, \varphi)$ be the corresponding intensity function for the sample (see Figure 2). We define the mean and standard deviation as follows

$$
\begin{gathered}
\mu_{r}=\frac{1}{4 \pi} \iint I_{r}(\theta, \varphi) \sin \varphi d \varphi d \theta, \\
\sigma_{r}^{2}=\frac{1}{4 \pi} \iint\left[I_{r}(\theta, \varphi)-\mu_{r}\right]^{2} \sin \varphi d \varphi d \theta .
\end{gathered}
$$

The condition for significant signal is then given by $\left|I_{p}(\theta, \varphi)-\mu_{r}\right|>5 \sigma_{r}$ and provides the means necessary to determine the fraction of the sphere deemed as useful for orientation determination, see Table 1 above. The total area of meaningful carbon signal in lysozyme increases with added water. While the opposite holds true in the case of sulfur, a $96 \%$ coverage in the $6 \AA$ case is still remarkable. This analysis suggests sulfur to be a superior candidate for orientation determination using explosion mapping.

To what extent can this approach be used to orient the sample molecule? To estimate the angular resolution obtainable with the explosion data we project the intensity functions onto spherical harmonics and calculate angular power spectra from the intensity coefficients. We define the power spectrum as

$$
P_{p ; l}=\sqrt{\sum_{m}\left|I_{p ; l m}\right|^{2}}
$$

where

$$
I_{p ; l m}=\iint I_{p}(\theta, \varphi) Y_{l}^{m *}(\theta, \varphi) \sin \varphi d \varphi d \theta
$$

is the intensity coefficient and $Y_{l}^{m *}(\theta, \varphi)$ is the complex conjugate of $Y_{l}^{m}(\theta, \varphi)$ - the spherical harmonic of degree $l$ and order $m$. The index $p$ (as shown above) refers to the sample, while $r$ is used for the random case.

Assuming a sampling rate of twice that of the angular resolution $\Delta \theta$ in accordance to the Nyquist-Shannon sampling theorem, the angular resolution from the explosion maps can be estimated as $\Delta \theta=2 \pi / l$. The highest degree where the power spectrum of the sample still is distinguishable from the random case is $l$, chosen as $\left|P_{p ; l}\right|>2\left|P_{r ; l}\right|$, when examining even and odd coefficients separately. Note that $\Delta \theta$ is the angular resolution given with respect to an equatorial line.

Table 2 below shows the results of the spherical harmonics analysis. As expected, the resolution is improved with a thicker water shell when measuring carbon ions. It is, however, still low for orientation purposes with an angular resolution of $40^{\circ}$ in the $6 \AA$-case. Sulfur is again proven to be the better option for orientation determination. In contrast to the carbon case, the angular resolution increases with the thickness of the water layer, and we predict an angular resolution better than $15^{\circ}$ for all cases. Our analysis has a cutoff at $12.4^{\circ}$, but from the analysis presented in Table 1 we can assume that the resolution is actually better than that (see also Supplementary Information, Figures 5 and 6). 
Table 2 Angular resolution from explosion maps of lysozyme, estimated by projection onto spherical harmonics. Highest degree indicates the largest value of $l$ where the condition $\left|P_{p ; l}\right|>2\left|P_{r, l}\right|$ still holds for functions of either even or odd parity. Our calculations are limited to a maximum degree of spherical harmonics at $l=29$, corresponding to an angular resolution of $12.4^{\circ}$.

\begin{tabular}{cccc} 
Element & Water layer & Highest degree & Angular resolution \\
\hline $\mathrm{C}$ & $0 \AA$ & $l=3$ & $120^{\circ}$ \\
$\mathrm{C}$ & $3 \AA$ & $l=5$ & $72^{\circ}$ \\
$\mathrm{C}$ & $6 \AA$ & $l=9$ & $40^{\circ}$ \\
$\mathrm{S}$ & $0 \AA$ & $l=25$ & $14.4^{\circ}$ \\
$\mathrm{S}$ & $3 \AA$ & $l>29$ & $<12.4^{\circ}$ \\
$\mathrm{S}$ & $6 \AA$ & $l>29$ & $<12.4^{\circ}$
\end{tabular}

Another relevant aspect of the explosion reproducibility is the behavior of individual ions. By quantifying the angular spread of each sample atom we can determine which atoms have the most consistent trajectories. If we represent the directionality of an ejected ion $i$ in explosion $k$ by a unit vector $\vec{v}_{i k}$, one such measure can be defined as the magnitude of the mean vector over all explosions,

$$
R_{i}=\left|\frac{1}{M} \sum_{k=1}^{M} \vec{v}_{i k}\right| .
$$

The resulting variable will take on a real value between 0 and $1, R_{i} \in[0,1]$, where 1 indicates a perfectly consistent flight direction (no spread) and 0 indicates an irreproducible, random one. Note that the lower limit is reached only for an infinite number of simulated explosions, i.e. $\lim _{k \rightarrow \infty} R_{i}=0$, for a fully reproducible atom. In some instances, such as if a particle has two distinct possible trajectories with little spread within them, the $R_{i}$-value would fail to capture the consistency of each direction and instead recognize it as a single hot spot with lower reproducibility. However, we assume such false negatives to be rare and their impact negligible to our analyses.

With this measure in place, we compared the carbon $R_{i}$-values in waterless lysozyme in correlation to their initial distance from the protein center of mass, their associated amino acids, and their placement in the polypeptide chain. In the first case we found no significant correlation, indicating that the distance to the center of mass does not affect the reproducibility of the trajectories. Considering the earlier suggestion of a shielding mechanism this seems somewhat surprising, but could be explained by the modest size of the sample. Moreover, since this analysis only covers the waterless variant of the sample, the potential shielding of water is disregarded. A similar conclusion can be drawn when grouping the carbons by their associated amino acids and comparing the mean $R_{i}$-values between the 20 different groups. No particular amino acid seems to correlate to a higher or lower reproducibility of carbon trajectories. Figures 7 and 8 in Supplementary Information show the data from both these tests.

However, when examining reproducibility in relation to the sequence in which the carbons are arranged along the polypeptide chain, we found that significant drops in $R_{i}$ appear in clusters (see Figure 9, Supplementary Information). This indicates that certain sections of the chain give carbons a greater angular freedom during the explosion, and it is neither correlated to how deep into the protein this segment is located, nor to the specific amino acid species which it consists of.

\section{Discussion and Conclusion}

We have found that, given the model used here, all our systems clearly exhibit explosion reproducibility to varying degrees. When examining carbon and sulfur, the ion density maps consistently produce a signal dissimilar to the map of a fully random distribution in both cases. Carbon trajectories are influenced substantially by the addition of water, seemingly through a shielding mechanism where the water molecules collectively cut off certain escape paths. Contrary to this, the heavier sulfurs display highly consistent explosion patterns almost unaffected by the different water layers, making them particularly interesting for orientation determination. The applicability of the approach presented here to experimental circumstances remain challenging however, as retrieving the full ion density across all spatial angles may prove troublesome due to physical space limitations, detector capacities, sufficient power supply etc. An alternative idea could instead be to sample partial explosion maps and use the data in conjunction with collected X-ray diffraction patterns. Such hybrid method would likely attain higher efficiency and accuracy compared to the particle orientation process used today. Estab- 
lishing the feasibility of a hybrid setup would require investigation beyond the scope of this study, but our findings suggests that retrieving even a map with partial coverage could provide valuable information about the orientation of the molecule at the time of X-ray interaction. The fact that carbon ion maps of samples with greater water coverage seem to show especially reproducible patterns is of particular interest for mainly two reasons. Firstly, all proteins contain carbon in large amounts (as opposed to sulfur) meaning there would always be a clear signal to measure. Secondly, surface-bound water is frequently present under experimental conditions, so seeing how it actually improves explosion reproducibility could prove to be beneficial. The question remains however if adding significantly more water, such as in bigger droplets or even in a liquid jet, would enhance the reproducibility to an even greater extent. If so, this idea could potentially be expanded to those setups as well. In cases where water content is variable and unknown, the carbon ion maps may also be useful for determining water content and detecting spatial inhomogeneous distribution of the water around the molecule. This information could provide additional assistance to data analysis methods that seek to resolve heterogeneous structural variation.

Because atoms of the same type - for example all carbons - were treated as indistinguishable from each other, the resulting ion density maps are actually the combined maps of each of the individual carbon atoms. Handling individual maps separately could theoretically be more useful for the purpose of spatial orientation, as then one could select the carbons with most consistent trajectory paths and disregard the rest. This would artificially increase the explosion reproducibility and, in turn, improve orientation accuracy. While it is clearly not realistically applicable in the normal case, a similar idea would be to substitute a few atoms of the sample with heavier elements (either distinguishable by their atomic weight or by their flight directions) and screen for those following the explosion. This situation would be akin to detecting sulfur ions of Lys, but implementable regardless of sample composition. The consistent trajectories of sulfurs in Lys inspires another inquiry, namely if this observation is generalizable to any sample, heavier element, or both. If so, it would not only strengthen the substitution approach described above, but also enable explosion mapping for spatial orientation of most proteins without atomic modifications. While it is likely that such correlation would not be quite so straightforward, perhaps additional refinements could be made by also simultaneously examining the influence of other factors not considered here, such as the local structures around the heavy atoms. For instance, when assessing the sulfur ions from Lys individually we found a convincing difference in explosion path variance between the sulfurs of methionine and those of cysteine, with the latter showing greater trajectory consistencies. All of the cysteine sulfurs also form pairwise disulfide bonds, which likely contributes to their explosion reproducibility due to the accumulation of greater local Coulomb forces. The methionine sulfurs do not form such bonds however, and since they bind to hydrophobic side-chains they tend to embed deeper inside the protein. This would presumably cause their trajectories to be less consistent. See Figure 4 for details on the placement and explosion behavior of these atoms.

It is worth noting that our simulations are done on non-symmetric proteins. If there is symmetry within the protein we expect the ion maps to exhibit this symmetry in similar way to diffraction patterns from the same protein. Orientation recovery from the ion explosion will hence be limited by the symmetry of the protein.

The current study is limited to looking at one set of pulse parameters. However, both the explosion and the diffracted images are dependent on the properties of the X-ray pulse, and position of the sample in relation to the focus of the X-ray beam. The temporal profile of the pulses from an XFEL are known to vary on shot-to-shot basis ${ }^{31,32}$, and the temporal profile has been shown to have impact on the ionization dynamics in the sample ${ }^{33}$. This is a problem that current protein crystallography experiments deal with by simply dismissing the diffraction images where the Bragg peaks are impossible to index. In general, the explosion dynamics is expected to be more reproducible when exposed to short X-ray pulses than to longer pulses, assuming that the total number of photons hitting the sample is the same. In the case of a long pulse, the dynamics of the Coulomb explosion is slower and the trajectories of the ions are less well defined.

It has been suggested in an earlier experimental study on imaging and explosion of aerosolized particles ${ }^{34}$, to use ion fragmentation as an indication for an XFEL pulse hitting the particle. With the repetition rates of the new European XFEL at 27000 pulses per second and expected diffraction data rates of $100 \mathrm{~GB} / \mathrm{s}^{35}$, it is necessary to identify which diffraction data is worth saving, and a detection of the fast hydrogen ions could offer such a solution. It could also be worthwhile to measure the plasma emission spectra in parallel with the diffracted image and the ion data, as a way to detect if the data from a hit is usable ${ }^{36}$. In particular, X-ray emission from the sulfurs in the sample was found to be useful in identifying when the sample has been hit by the X-ray pulse.

A recent study analyzed the improvement in orientation recovery by aligning the samples in an electric field during exposure to an XFEL pulse ${ }^{37}$. An enhanced version of the EMC algorithm (EEMC) that takes advantage of prior knowledge 

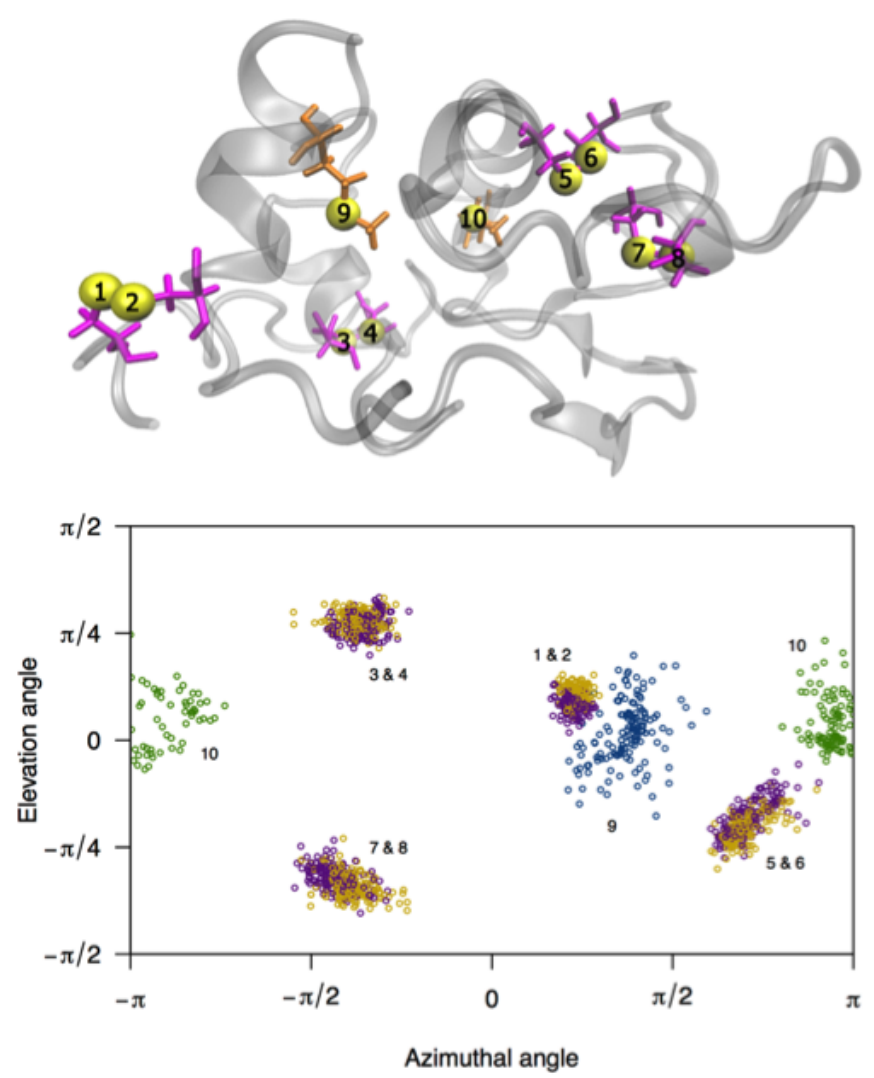

Fig. 4 The sulfur atoms of lysozyme. [top] The Lys molecule without surrounding water, with amino acids cysteine highlighted in purple and methionine in orange. Sulfur atoms are labeled and shown as yellow spheres. The cysteine sulfurs form pairwise disulfide bonds while the sulfurs of methionine remain separated. [bottom] The sulfur ion distribution across all 150 explosions of Lys with no water, using the same sulfur labels. Paired cysteine sulfurs have similar trajectories with less angular variation in comparison to the isolated methionine sulfurs. As an example, sulfur 1 has a standard deviation of $4.2^{\circ}$ in azimuthal angle and $3.6^{\circ}$ in elevation angle, while the corresponding numbers for sulfur 9 are $12.2^{\circ}$ and $13.1^{\circ}$.

about the orientation was implemented. The tests were carried out on a protein oriented to a resolution of $7^{\circ}$ in one dimension, i.e. only the direction of one axis of the protein was known. The study showed that (i) knowledge about orientation speeds up the EMC algorithm substantially, and (ii) sample orientation could be recovered even in cases where it was deemed impossible, for example where the diffraction data was too noisy or where some data was missing due to the experimental geometry. Taking advantage of the reproducibility of the explosion, as is presented here, would give the orientation of the protein up to a high resolution along both angular axes and further improve the capabilities of the EEMC approach. From Table 2 we estimate an orientation using the sulfur signal to an angular resolution lower than $15^{\circ}$ along both the elevation and azimuthal axes. This is an orientation more well defined that what described in Marklund et al. ${ }^{37}$, where the sample is oriented up to $7^{\circ}$ along one of its axes, but free to rotate $360^{\circ}$ around the same axis.

In conclusion, this proof-of-concept work shows that the Coulomb explosion of a biomolecule is sufficiently reproducible to encode information about spatial orientation that can be recorded in an angular ion map. This information may be useful for methods of determining molecular orientation through data analysis. Surrounding water has a significant influence on the carbon ion trajectories, while the heavier sulfurs (in the case of Lys) are less affected. Therefore, explosion mapping of heaver ions might be preferable in circumstances where the details of the hydration shell are unknown. We expect this knowledge to be of significant importance in single particle imaging techniques as they continue to develop.

\section{Acknowledgements}

We thank the Swedish Research Foundation for Strategic Research, the Swedish Research Council (2013-3940), the Swedish Research Council via the Röntgen-Ångström Cluster, the Carl Trygger Foundation, the Helmholtz Association through the Center for Free-Electron Laser Science at DESY and the Australian Research Council's Centre of Excellence 
Programme. Gratitude also goes out to the Swedish National Infrastructure for Computing (SNIC) through Uppsala Multidisciplinary Center for Advanced Computational Science (UPPMAX) under project snic2016-7-15 and the Swedish Foundation for International Cooperation in Research and Higher Education (STINT). Finally, we want to thank Erik Marklund and Davide Ragazzon for help and support with the simulations and the analyses.

\section{References}

1 J. Westbrook, Z. Feng, S. Jain, T. N. Bhat, N. Thanki, V. Ravichandran, G. L. Gilliland, W. Bluhm, H. Weissig, D. S. Greer, P. E. Bourne, and H. M. Berman, Nucleic Acids Res., 2002, 30, 245-248.

2 R. Neutze, R. Wouts, D. van der Spoel, E. Weckert and J. Hajdu, Nature, 2000, 406, 752-757.

3 M. M. Seibert, S. Boutet, M. Svenda, T. Ekeberg, F. R. N. C. Maia, M. J. Bogan, N. Timneanu, A. Barty, S. Hau-Riege, C. Caleman, M. Frank, H. Benner, J. Y. Lee, S. Marchesini, J. W. Shaevitz, D. A. Fletcher, S. Bajt, I. Andersson, H. N. Chapman and J. Hajdu, J. Phys. B: At. Mol. Opt. Phys, 2010, 43, 194015.

4 T. E. Ekeberg, M. Svenda, C. Abergel, F. R. N. C. Maia, V. Seltzer, J.-M. Claverie, M. Hantke, O. Jönsson, C. Nettelblad, G. van der Schot, M. Liang, D. P. DePonte, A. Barty, M. M. Seibert, B. Iwan, I. Andersson, N. D. Loh, A. V. Martin, H. Chapman, C. Bostedt, J. D. Bozek, K. R. Ferguson, J. Krzywinski, S. W. Epp, D. Rolles, A. Rudenko, R. Hartmann, N. Kimmel and J. Hajdu, Phys. Rev. Lett., 2015, 114, 098102.

5 A. Aquila, A. Barty, C. Bostedt, S. Boutet, G. Carini, D. dePonte, P. Drell, S. Doniach, K. H. Downing, T. Earnest, H. Elmlund, V. Elser, M. Guhr, J. Hajdu, J. Hastings, S. P. Hau-Riege, Z. Huang, E. E. Lattman, F. R. N. C. Maia, S. Marchesini, A. Ourmazd, C. Pellegrini, R. Santra, I. Schlichting, C. Schroer, J. C. H. Spence, I. A. Vartanyants, S. Wakatsuki, W. I. Weis and G. J. Williams, Struct. Dyn., 2015, 2, 041701.

6 N.-T. D. Loh and V. Elser, Phys. Rev. E, 2009, 80, 026705.

7 P. Schwander, R. Fung, G. Phillips and A. Ourmazd, New J. Phys., 2010, 12, 35007.

8 S. P. Hau-Riege, R. A. London and A. Szőke, Phys. Rev. E, 2004, 69, 051906.

9 Z. Jurek, G. Faigel and M. Tegze, Eur. Phys. J. D, 2004, 29, 217-229.

10 M. Bergh, N. Tîmneanu and D. van der Spoel, Phys. Rev. E, 2004, 70, 051904.

11 Z. Jurek and G. Faigel, Europhys. Lett., 2013, 101, 16007.

12 J. W. Neidigh, R. M. Fesinmeyer and N. H. Andersen, Nature Struct. Biol., 2002, 9, 425 - 430.

13 M. Leijonmarck and A. Liljas, J. Mol. Biol., 1987, 195, 555.

14 S. Vijay-Kumar, C. E. Bugg and W. J. Cook, J. Mol. Biol., 1987, 194, 531-544.

15 P. J. Artymiuk, C. F. Blake, D. W. Rice and K. S. Wilson, Acta Crystallogr. Sect. B, 1982, 38, 778.

16 A. Patriksson, E. Marklund and D. van der Spoel, Biochemistry, 2007, 46, 933-945.

17 E. G. Marklund, D. S. D. Larsson, D. van der Spoel, A. Patriksson and C. Caleman, Phys. Chem. Chem. Phys., 2009, 11, 8069-8078.

18 R. Neutze, G. Huldt, J. Hajdu and D. van der Spoel, Radiat. Phys. Chem., 2004, 71, 905-916.

19 C. Caleman, G. Huldt, C. Ortiz, F. R. N. C. Maia, F. G. Parak, J. Hajdu, D. van der Spoel, H. N. Chapman and N. Tîmneanu, ACS Nano, 2011, 5, 139-146.

20 C. Caleman, M. Bergh, H. A. Scott, J. C. H. Spence, H. N. Chapman and N. Tîmneanu, J. Mod. Opt., 2011, 58, 14861497.

21 D. van der Spoel, E. Lindahl, B. Hess, G. Groenhof, A. E. Mark and H. J. C. Berendsen, J. Comp. Chem., 2005, 26, 1701-1718.

22 W. L. Jorgensen and J. Tirado-Rives, J. Am. Chem. Soc., 1988, 110, 1657-1666.

23 G. A. Kaminski, R. A. Friesner, J. Tirado-Rives and W. L. Jorgensen, J. Phys. Chem. B, 2001, 105, 6474-6487.

24 D. P. DePonte, U. Weierstall, K. Schmidt, J. Warner, D. Starodub, J. C. H. Spence and R. B. Doak, J. Phys. D, 2008, 41, 195505.

25 R. A. Kirian, S. Awel, N. Eckerskorn, H. Fleckenstein, M. Wiedorn, L. Adriano, S. Bajt, M. Barthelmess, R. Bean, K. R. Beyerlein, L. M. G. Chavas, M. Domaracky, M. Heymann, D. A. Horke, J. Knoska, M. Metz, A. Morgan, D. Oberthuer, N. Roth, T. Sato, P. L. Xavier, O. Yefanov, A. V. Rode, J. Küpper and H. N. Chapman, Struct. Dyn., 2010, 2, 41717.

26 M. J. Bogan, W. H. Benner, S. Boutet, U. Rohner, M. Frank, A. Barty, M. M. Seibert, F. Maia, S. Marchesini, S. Bajt, B. Woods, V. Riot, S. P. Hau-Riege, M. Svenda, E. Marklund, E. Spiller, J. Hajdu and H. N. Chapman, Nano Lett., 2008, 
8, 310-316.

27 W. L. Jorgensen, J. Chandrasekhar, J. D. Madura, R. W. Impey and M. L. Klein, J. Chem. Phys., 1983, 79, $926-935$.

28 P. M. Morse, Phys. Rev., 1929, 34, 57-64.

29 M. Liang, G. J. Williams, M. Messerschmidt, M. M. Seibert, P. A. Montanez, M. Hayes, D. Milathianaki, A. Aquila, M. S. Hunter, J. E. Koglin, D. W. Schafer, S. Guillet, A. Busse, R. Bergan, W. Olson, K. Fox, N. Stewart, R. Curtis, A. A. Miahnahri and S. Boutet, J. Synchrotron Radiat., 2015, 22, 514-519.

30 S. Krinsky and R. L. Gluckstern, Phys. Rev. Accel. Beams, 2003, 6, 50701.

31 C. Behrens, F.-J. Decker, Y. Ding, V. A. Dolgashev, J. Frisch, Z. Huang, P. Krejcik, H. Loos, A. Lutman, T. J. Maxwell, J. Turner, J. Wang, W. M.-H., J. Welch and J. Wu, Nat. Comm., 2014, 4, 3762.

32 W. Helml, A. R. Maier, W. Schweinberger, I. Grguraš, P. Radcliffe, G. Doumy, C. Roedig, J. Gagnon, M. Messerschmidt, S. Schorb, C. Bostedt, F. Grüner, L. F. DiMauro, D. Cubaynes, J. D. Bozek, T. Tschentscher, J. T. Costello, M. Meyer, R. Coffee, S. Düsterer, A. L. Cavalieri and R. Kienberger, Nat. Photonics, 2014, 8, 950-957.

33 H. O. Jönsson, N. Tîmneanu, C. Östlin, H. A. Scott and C. Caleman, J. Synchrotron Radiat., 2015, 22, 256-266.

34 J. Andreasson, A. V. Martin, M. Liang, N. Timneanu, A. Aquila, F. Wang, B. Iwan, M. Svenda, T. Ekeberg, M. Hantke, J. Bielecki, D. Rolles, A. Rudenko, L. Foucar, R. Hartmann, B. Erk, B. Rudek, H. N. Chapman, J. Hajdu and A. Barty, Opt. Express, 2014, 22, 2497-2510.

35 F. R. N. C. Maia and J. Hajdu, Sci. Data., 2016, 3, 160059.

36 H. O. Jönsson, C. Caleman, J. Andreasson and N. Tîmneanu, IUCrJ, 2017, 4,.

37 E. G. Marklund, T. Ekeberg, M. Moog, J. L. P. Benesch and C. Caleman, J. Phys. Chem. Lett., 2017, 8, 4540-4544. 